\title{
The Measurement of Amino Groups in Proteins and Peptides
}

\author{
BY ROBERT FIELDS \\ Department of Biochemistry, University of Cambridge, Tennis Court Road, Cambridge CB2 1QW, U.K.
}

(Received 7 May 1971)

\begin{abstract}
A technique is examined for determining amino groups with 2,4,6-trinitrobenzenesulphonic acid, in which the extinction at $420 \mathrm{~nm}$ of sulphite complexes of the trinitrophenylated amino groups is measured. The sensitivity of the method is 5-200 nmol of amino group. The method is especially suitable for checking the extent of blocking or unblocking of amino groups in proteins and peptides, owing to the short time required for reaction ( $5 \mathrm{~min}$ at room temperature). The reaction of the reagent with thiol groups has been studied and was found to proceed 30-50 times faster than with $\epsilon$-amino groups of model compounds. The $\epsilon_{420}$ of a trinitrophenylated thiol group was found to be $2250 \mathrm{M}^{-1} \cdot \mathrm{cm}^{-1}$. The reaction with several amino acids, peptides and proteins is presented. The $\epsilon_{420}$ of a typical $\alpha$-amino group was found to be $22000 \mathrm{M}^{-1} \cdot \mathrm{cm}^{-1}$ and that of an $\epsilon$-amino group, $19200 \mathrm{M}^{-1} \cdot \mathrm{cm}^{-1}$. Difficulties inherent in the analysis of constituent amino group reactions in proteins are discussed.
\end{abstract}

Since Okuyama \& Satake (1960) proposed the use of TNP-sulphonic acid* as a reagent specific for primary amino groups several techniques have been described for determining amino groups or for studying the rate of reaction of TNP-sulphonic acid with amino groups in proteins (Satake, Okuyama, Ohashi \& Shinoda, 1960; Habeeb, 1966; Goldfarb, $1966 a$; Freedman \& Radda, 1968). Ribonuclease $\mathrm{T}_{1}$ (EC 2.7.7.26), which has two amino groups, one of which may be selectively removed (Fields \& Dixon, 1971) was initially used as a substrate to investigate whether complete reaction of TNPsulphonic acid could be achieved within 5-10 min that would also permit kinetic analysis to be made. The study was then extended to try to define more clearly the use of TNP-sulphonic acid as a reagent for mapping areas of nucleophilic reactivity in enzymes. The procedure that was devised with ribonuclease $T_{1}$ for following reaction rates was altered to make it convenient as a general method for determining amino groups. Both procedures make use of the phenomenon observed by Goldfarb (1966a) of the reversible association of sulphite with TNP-amino groups to form complexes which have an absorption maximum near $420 \mathrm{~nm}$. As this wavelength is removed from the region of absorption of TNPsulphonic acid a high concentration of reagent may be used, with consequent shortening of the time required for reaction. The reaction is carried out in borate buffer at pH 9.5. At this $\mathrm{pH}$ TNP. sulphonic acid reacts with hydroxyl ions to give a

* Abbreviation: TNP-, 2,4,6-trinitrophenyl-. blank extinction. This side reaction is stopped by lowering the $\mathrm{pH}$ to neutrality after the amino groups have been trinitrophenylated (procedure 1; see the Materials and Methods section) or, if the rate of reaction of TNP-sulphonic acid with a sample is being studied, the blank extinction is continuously subtracted, by using a split-beam recording spectrophotometer (procedure 2).

\section{MATERIALS AND METHODS}

Reagents. TNP-sulphonic acid was obtained from BDH Chemicals Ltd., Poole, Dorset, U.K., and from Eastman Kodak Ltd., Kirkby, Liverpool, U.K. The quality of individual samples varied and, with one exception, required further purification before use. Samples of the tetrahydrate (BDH Chemicals Ltd.) or of the trihydrate (Eastman Kodak Ltd.) were dissolved in 1 part of water (w/w) by heating, and Anala $\mathrm{R} \mathrm{HCl}$ (sp.gr. 1.18) was then added to about $2 \mathrm{M}$. The solid that crystallized out on cooling was washed on the filter with cold $1 \mathrm{M}-\mathrm{HCl}$ before drying in a desiccator. The appearance of the dry reagent was flaky and white, resembling the trihydrate, and was unlike the large transparent yellow crystals of the tetrahydrate. A molecular weight of 347.2 was assumed. When sufficiently pure, TNP-sulphonic acid was found to have $\epsilon_{340}$ not greater than $600 \mathrm{M}^{-1} \cdot \mathrm{cm}^{-1}$. TNP-glycylglycine was prepared by treating a slight excess of glycylglycine with TNP-sulphonic acid in methanolic bicarbonate buffer. After $24 \mathrm{~h}$ the mixture was acidified, dried by rotary evaporation, and the residue extracted with ethyl acetate. The product was recrystallized from aqueous methanol. A solution of $N^{\alpha}$-acetyl-Llysine amide was a gift from Dr I. Gibbons and a sample of $N^{\alpha}$-acetyl-L-lysine was a gift from $\mathrm{Dr}$ H. B. F. Dixon. All other amino acids and their derivatives were 
of analytical grade and were used without further purification. Buffers and reagent solutions were made up in twice-distilled water that had been passed through a Permutit deionizer.

Proteins. Ribonuclease $\mathrm{T}_{1}$ was prepared from Taka Diastase by chromatography on DEAE-cellulose in a solution of $0.12 \mathrm{M}-\mathrm{Na}_{2} \mathrm{HPO}_{4}-0.08 \mathrm{M}-\mathrm{NaH}_{2} \mathrm{PO}_{4}$ (Fields, Dixon, Law \& Yui, 1971). Samples were transaminated by incubation for $30 \mathrm{~min}$ in a solution of $2 \mathrm{M}$-sodium acetate in $0.4 \mathrm{M}$-acetic acid that contained $0.1 \mathrm{M}$-glyoxylate and $10 \mathrm{~mm}$-cupric sulphate (Dixon, 1967). Ribonuclease $\mathbf{T}_{1}$, so treated, showed one reactive carbonyl group per molecule, as determined with 2,4-dinitrophenylhydrazine (Fields \& Dixon, 1971), and was more retarded on DEAEcellulose chromatography than the native enzyme. Samples of ribonuclease $T_{1}$ and of transaminated ribonuclease $T_{1}$ were concentrated from the chromatographic buffer and gel-filtered into water, before allowing them to react with TNP-sulphonic acid. Ribonuclease A (EC 2.7.7.16) was prepared from Grade III pancreatic ribonuclease obtained from Miles-Seravac (Pty.) Ltd., Maidenhead, Berks., U.K., by chromatography on a column of CM-cellulose in $0.56 \mathrm{~m}$-acetate buffer, pH4.7. The main component, which emerged at 3 bed volumes, was concentrated and desalted by adsorption on to the acid form of CM-cellulose, followed by displacement with $25 \%$ (v/v) acetic acid (R. Fields \& H. B. F. Dixon, unpublished work). Ribonuclease A, obtained as a salt-free solid by freeze-drying from dilute acetic acid, was dissolved in water before allowing it to react with TNP-sulphonic acid. Solutions of insulin were prepared by dissolving crystalline bovine insulin (obtained from BDH Chemicals Ltd.) in dilute borate buffer, $\mathrm{pH} \mathrm{9.8,} \mathrm{before} \mathrm{use.}$

Standardization of samples. Solutions of $N^{\alpha}$-acetyl-Llysine amide, glycyl-L-lysine, ribonuclease $T_{1}$, transaminated ribonuclease $T_{1}$ and bovine insulin were standardized by determining the amount of lysine liberated after acid hydrolysis. Solutions in $6 \mathrm{M}-\mathrm{HCl}$ were hydrolysed at $108^{\circ} \mathrm{C}$ for $24 \mathrm{~h}$ in sealed evacuated tubes. The analyses were carried out on a Beckman model $120 \mathrm{C}$ amino acid analyser. The concentration of ribonuclease $A$ was estimated from its extinction at $280 \mathrm{~nm}$ by using the value $\epsilon 9600 \mathrm{~m}^{-1} \cdot \mathrm{cm}^{-1}$ (Sela \& Anfinsen, 1957).

Standardization of sulphite solutions. As it was not possible to stabilize sulphite solutions by adding EDTA (Cecil \& McPhee, 1955) all solutions were made up in deionized, twice-distilled water. The stability of sulphite solutions was checked by using $5,5^{\prime}$-dithiobis-(2-nitrobenzoic acid) and the procedure devised by Ellman (1959) for the determination of thiol groups. Sulphite ion is known to cleave disulphides (Clarke, 1932) and solutions of it were found to react quantitatively with Ellman's reagent in under $3 \mathrm{~min}$ (see Humphrey, Ward \& Hinze, 1970). As a standard curve was linear over the concentration range used, a molar extinction of $13600 \mathrm{M}^{-1} \cdot \mathrm{cm}^{-1}$ at $412 \mathrm{~nm}$ for the anion was assumed (Ellman, 1959). A stock solution of $0.1 \mathrm{~m}-\mathrm{Na}_{2} \mathrm{SO}_{3}$ was observed to decrease in concentration by about $10 \%$ in $24 \mathrm{~h}$ and by $3-5 \%$ daily thereafter.

Effect of sulphite on the extinction of TNP-glycylglycine and $T N P$-sulphonic acid. Goldfarb (1966a) reported that $\mathrm{Na}_{2} \mathrm{SO}_{3}$ decreased the extinction at $345 \mathrm{~nm}$ and increased the extinction at $420 \mathrm{~nm}$ of TNP-amino acids; however, higher concentrations of sulphite appeared to cause a

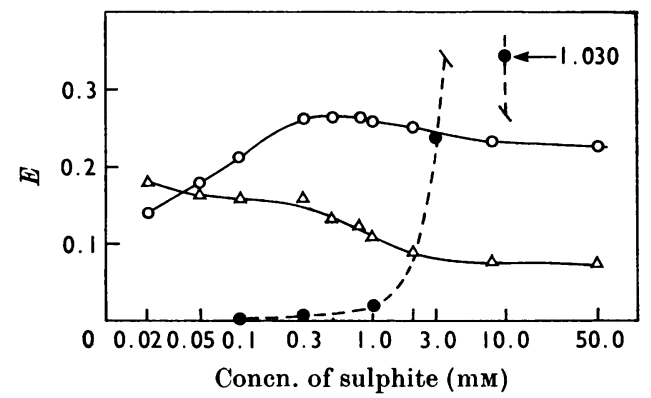

Fig. 1. Effect of sulphite on the extinction of TNPglycylglycine and TNP-sulphonic acid. Solutions containing approx. $12 \mu \mathrm{M}$-TNP-glycylglycine or $10 \mathrm{mM}$-TNPsulphonic acid in 0.1 M-pyrophosphate buffer, $\mathrm{pH} 8.5$, were made up in $\mathrm{Na}_{2} \mathrm{SO}_{3}$ to the concentrations shown, before the extinction at $340 \mathrm{~nm}$ or $420 \mathrm{~nm}$ was determined. $\triangle, E_{340}$ of TNP-glycylglycine; $\bigcirc, E_{420}$ of TNP-glycylglycine; $\bullet, E_{420}$ of TNP-sulphonic acid.

decrease in extinction at $420 \mathrm{~nm}$. To investigate this relationship, solutions containing TNP-glycylglycine and various concentrations of $\mathrm{Na}_{2} \mathrm{SO}_{3}$ were prepared in $50 \mathrm{~mm}$ pyrophosphate buffer, $\mathrm{pH}$ 8.5. Fig. 1 shows the extinctions of these solutions at $340 \mathrm{~nm}$ and at $420 \mathrm{~nm}$.

It was observed that solutions of TNP-sulphonic acid and $\mathrm{Na}_{2} \mathrm{SO}_{3}$, when concentrated, possessed a crimson colour at alkaline $\mathrm{pH}$. This was found to be associated with the appearance of a new extinction maximum at $490 \mathrm{~nm}$. Since the crimson colour disappeared on acidification or dilution, it was assumed that this colour arose from the formation of a complex between sulphite and TNP-sulphonic acid, analogous to that formed with TNP-amino acids. Fig. 1 shows the increase in extinction at $420 \mathrm{~nm}$ that occurs in a solution of $10 \mathrm{~mm}$-TNP. sulphonic acid as the concentration of sulphite is increased.

Preparation of the TNP-sulphonic acid solution and buffers. (1) TNP-sulphonic acid. A $1.8 \mathrm{M}$-solution of TNP-sulphonic acid was prepared by accurately weighing $100-500 \mathrm{mg}$ of TNP-sulphonic acid and adding $0.1 \mathrm{ml}$ of deionized water for each $100 \mathrm{mg}$ of reagent. The crystals were dissolved by gentle warming and the pale-yellow solution was kept stoppered. Picric acid, which absorbs at $420 \mathrm{~nm}$ in the presence of sulphite, is slowly formed in this solution, but solutions that were stored frozen were used daily for a week without great deterioration. (2) Buffers. A solution of $0.1 \mathrm{M}-\mathrm{Na}_{2} \mathrm{~B}_{4} \mathrm{O}_{7}$ in $0.1 \mathrm{~m}-\mathrm{NaOH}$ was used for carrying out the trinitrophenylations. The reaction was stopped by adding $0.1 \mathrm{M}-\mathrm{NaH}_{2} \mathrm{PO}_{4}$, which contained approx. $1.5 \mathrm{~mm}-\mathrm{Na}_{2} \mathrm{SO}_{3}$ to increase the extinction of the TNP-amino groups that were formed (Fig. 2). This solution was made up daily by adding $1.5-2 \mathrm{ml}$ of $0.1 \mathrm{M}-\mathrm{Na}_{2} \mathrm{SO}_{3}$ stock solution to $100 \mathrm{ml}$ of $0.1 \mathrm{M}-\mathrm{NaH}_{2} \mathrm{PO}_{4}$, the actual sulphite concentration being checked afterwards, if necessary by the Ellman procedure. The concentration of sulphite in the $0.1 \mathrm{~m}$-phosphate solution was never found to decrease by more than $20 \%$ in $8 \mathrm{~h}$; hence the effect of this decrease on the assay of TNP-amino groups was negligible (Fig. 2). 
Procedure 1. Determination of amino groups. A sample that contains amino groups is added to $0.5 \mathrm{ml}$ of borate buffer $\left(0.1 \mathrm{M}-\mathrm{Na}_{2} \mathrm{~B}_{4} \mathrm{O}_{7}\right.$ in $\left.0.1 \mathrm{M}-\mathrm{NaOH}\right)$ and the volume

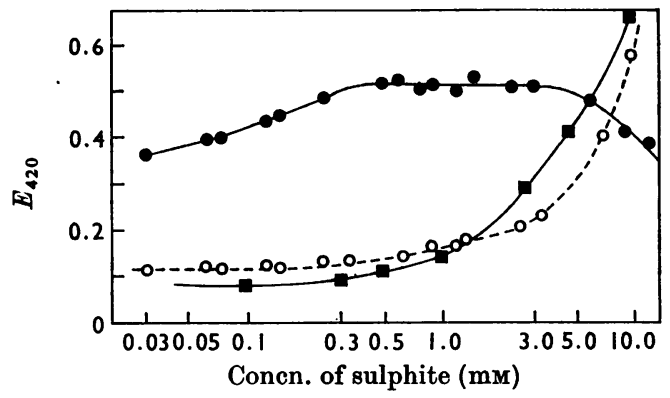

Fig. 2. Effect of sulphite on the extinction at $420 \mathrm{~nm}$ of the reaction product of L-lysine or $N$-acetyl-L-cysteine and TNP-sulphonic acid after incubation for $5 \mathrm{~min}$ at $\mathrm{pH} 9.5$. A final concentration of $45 \mu \mathrm{M}$-lysine or $180 \mu \mathrm{M}-N$-acetylL-cysteine reacted with $36 \mathrm{~mm}$-TNP-sulphonic acid. The reactions were stopped after $5 \mathrm{~min}$ by adding $2.0 \mathrm{ml}$ of $0.1 \mathrm{M}-\mathrm{NaH}_{2} \mathrm{PO}_{4}$ to which had been added an amount of $\mathrm{Na}_{2} \mathrm{SO}_{3}$ to give the final concentrations shown. Sulphite concentration was determined in each case by using $5,5^{\prime}$-dithiobis-(2-nitrobenzoic acid). $\bullet, E_{420}$ of solutions that contained L-lysine, minus the extinction of a blank incubated under the same conditions; $\square, E_{420}$ of solutions that contained $N$-acetyl-L-cysteine, minus blank extinction; $O, E_{420}$ of blank solutions. is made up to $1.0 \mathrm{ml}$. Then, $20 \mu \mathrm{l}$ of $1.8 \mathrm{M}$-TNP-sulphonic acid solution is added and the solution rapidly mixed. After $5.0 \mathrm{~min}$ the reaction is stopped by adding $2.0 \mathrm{ml}$ of $0.1 \mathrm{M}-\mathrm{NaH}_{2} \mathrm{PO}_{4}$ that contains $1.5 \mathrm{~mm}$-sulphite and the extinction at $420 \mathrm{~nm}$ is determined. A blank is also prepared. When multiple determinations are made, it is convenient to add TNP-sulphonic acid by microsyringe to successive samples at $15 \mathrm{~s}$ intervals, mixing the contents of the tubes between additions. The reactions are stopped after $5 \mathrm{~min}$ in the same order at $15 \mathrm{~s}$ intervals, by adding the phosphate solution from a repeating $2.0 \mathrm{ml}$ pipette. Table 1 shows the molar extinctions of compounds determined by this procedure.

Procedure 2. Determination of the rate of reaction of $T N P$-sulphonic acid with amino groups. To a solution of amino groups $(2.0 \mathrm{ml})$ in $0.05 \mathrm{M}-\mathrm{Na}_{2} \mathrm{~B}_{4} \mathrm{O}_{7}$ in $0.5 \mathrm{M}-\mathrm{NaOH}$ in a cuvette of $1 \mathrm{~cm}$ light-path length is added $20 \mu \mathrm{l}$ of $0.1 \mathrm{M}-\mathrm{Na}_{2} \mathrm{SO}_{3}$; this addition is also made to a cuvette containing $2.0 \mathrm{ml}$ of buffer. Then, $20 \mu \mathrm{l}$ of $1.8 \mathrm{M}$-TNPsulphonic acid solution is added to each cuvette. The solutions are rapidly mixed by inversion and placed in the sample and reference compartments of a split-beam recording spectrophotometer, and the differences in extinction at $420 \mathrm{~nm}$ are recorded. Fig. 3 shows curves that were produced on a Beckman DK 2 spectrophotometer from the reaction of TNP-sulphonic acid with ribonuclease $T_{1}$ and with transaminated ribonuclease $T_{1}$. Table 2 shows the rate of reaction of the reagent with various compounds, determined from curves produced in this way.

Analysis of the curves. When the reaction has stopped (as evidenced by no further increase in extinction) the

Table 1. Molar extinction coefficients at $420 \mathrm{~nm}$, determined by procedure 1

Values for constituent $\alpha$-and $\epsilon$-amino groups were estimated by using procedure 2 as described in the text.

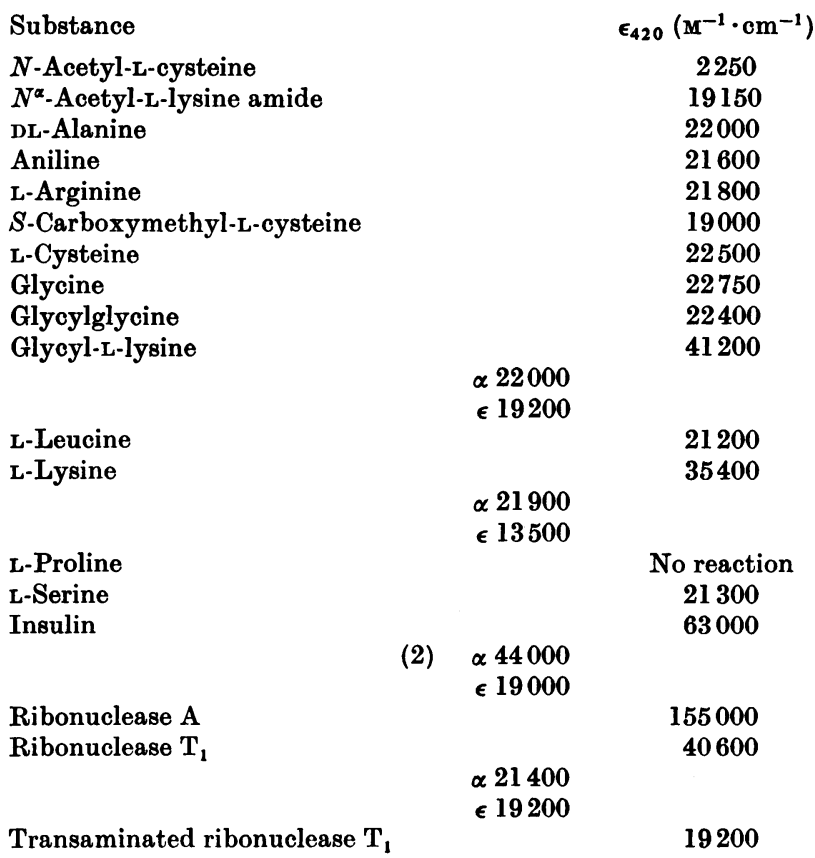




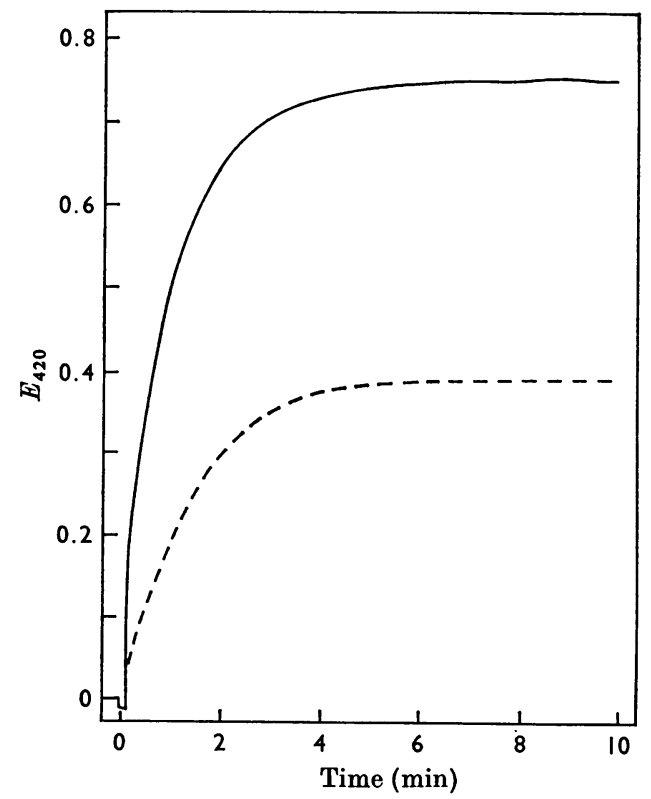

Fig. 3. Reaction of TNP-sulphonic acid (18 mM) with ribonuclease $\mathrm{T}_{1}(18 \mu \mathrm{M})$ and with transaminated ribonuclease $\mathrm{T}_{1}(19 \mu \mathrm{M})$. The reactions were carried out according to procedure 2 in the text. The original curves produced by the spectrophotometer were drawn from right to left. $\longrightarrow$, Ribonuclease $T_{1} ;----$, transaminated ribonuclease $T_{1}$.

curves may be analysed by the method of Goldfarb $(1966 b)$, or in the case of compounds with only one amino group, the half-life may be obtained simply by inspection of the curve. Since the concentration of TNP-sulphonic acid greatly exceeds that of amino groups, the reaction was assumed to be pseudo-first-order for each class of amino group. The total concentration of amino groups was calculated from the final extinction at $420 \mathrm{~nm}$ of the trinitrophenylated sample $\left(\Delta E_{\mathrm{final}}\right)$. The concentration of amino groups at a particular time was obtained by subtracting the extinction of the reacted amino groups at that time $\left(\Delta E_{t}\right)$ from the final extinction $\left(\Delta E_{\text {final }}\right)$. Several values of $\Delta E_{\text {final }}-\Delta E_{t}$ were plotted semi-logarithmically and the half-life for the reaction was determined, in the case of a single class of reacting amino groups, from the best-fit straight line. When there were two amino groups in the sample (e.g. ribonuclease $\mathrm{T}_{1}$, glycyl-L-lysine), the final slope attained by $\log \left(\Delta E_{\text {final }}-\Delta E_{t}\right)$ was extrapolated to the $y$-axis and values of $\Delta E_{\mathrm{final}}-\Delta E_{t}$ in the extrapolated line were subtracted from corresponding values in the original curve. These difference values were plotted semilogarithmically; if they were in a straight line, the two lines were taken to represent constituent reactions, and the intercept of each line with the $y$-axis permitted the molar extinction of each amino group to be determined. If the second set of values deviated from a straight line, the first line was redrawn, and the procedure repeated until two straight lines were obtained (Figs. 4 and 5). If the second set of values could not be made to fit a straight line, its final slope was extrapolated to the $y$-axis and values of $\Delta E_{\text {final }}-\Delta E_{t}$ were subtracted from corresponding values in the second curve. These difference values were then plotted as before.

Reaction of TNP-sulphonic acid with L-cysteine and $\mathrm{N}$-acetyl-L-cysteine. Owing to the rapid rate of reaction of TNP-sulphonic acid with thiol groups (Table 2) TNPsulphonic acid was diluted 200 -fold before use; only the initial portions of the curves, which obeyed pseudo-firstorder rate laws, were plotted as $\log \left(\Delta E_{\mathrm{final}}-\Delta E_{t}\right)$. The TNP-thiol-sulphite complex was found to have an extinction maximum at $455 \mathrm{~nm}$; its molar extinction at $420 \mathrm{~nm}$ is shown in Table 1 , as this wavelength is relevant to the assay.

\section{RESULTS AND DISCUSSION}

Assay. Procedure 1. The slowest reacting amino groups studied by procedure 2 possessed half-lives in the range 50-84s (Table 2). As the concentration of TNP-sulphonic acid used in procedure 1 is twice that used for making these determinations, the slowest reacting group found, namely the $\epsilon$ amino group of lysine, would be expected to undergo more than $99 \%$ reaction in $5 \mathrm{~min}$. Still, in some circumstances it may be desirable to extend the incubation period to 10 or $20 \mathrm{~min}$. This can be done without greatly affecting the accuracy of the assay as the blank extinction at $420 \mathrm{~nm}$ increases linearly at a rate of about $0.018 / \mathrm{min}$. Fig. 2 shows that the extinction of blanks increases as sulphite is added and increases rapidly when the concentration is greater than $3-5 \mathrm{~mm}$. This increase is due to the formation of TNP-sulphonic acid-sulphite complexes (Fig. 1). The pink colour imparted to blank solutions by mM-sulphite is a convenient indication that the concentration of sulphite is adequate.

Procedure 2. The blank extinction at pH 9.5 is stimulated markedly by light. An initial rate of $0.040 / \mathrm{min}$ for procedure 2 was observed in the Beckman DK 2 spectrophotometer used in this study. This light-induced increase in extinction is greater at the beginning of the reaction than later on; nevertheless its course is reproducible and differences in extinction between two blank solutions in $0.5 \mathrm{~h}$ are usually small. The solution of 1.8 M-TNP-sulphonic acid was carefully added to the bottom of both blank and sample cuvettes, before mixing, with the same microsyringe to minimize differences in the blank rate. Both solutions were then mixed simultaneously and placed in the instrument, or the sample solution was mixed seconds after the blank. The solution of $1.8 \mathrm{M}$ TNP-sulphonic acid is viscous, and requires vigorous mixing by inversion of the cuvette to effect complete dispersion.

The $\epsilon_{420}$ values of TNP-amino group-sulphite 
Table 2. Rate of reaction of TNP-sulphonic acid with various compounds

The rate was determined by procedure 2 (18 mM-TNP-sulphonic acid, $1 \mathrm{~mm}-\mathrm{Na}_{2} \mathrm{SO}_{3}, 50 \mathrm{~mm}$-borate buffer, pH 9.5).

Substance

$N$-Acetyl-L-cysteine

L-Cysteine

$S$-Carboxymethyl-L-cysteine

Glycine

Glycylglycine

$N^{\alpha}$-Acetyl-L-lysine amide

L-Alanine

$N^{\alpha}$-Acetyl-L-lysine

Transaminated ribonuclease $\mathrm{T}_{1}$ L-Lysine

Glycyl-L-lysine

Ribonuclease $\mathbf{T}_{\mathbf{s}}$

Insulin

Ribonuclease A

Number of groups/kinetic set

(1) 2.6

(2)* 1.0

(3) 2.4

Total 8.0

$\begin{array}{ccc}\text { Amino group } & \text { Half-life }(\mathrm{s}) & \begin{array}{c}\text { Second-order rate } \\ \text { constant }\left(k_{2}\right)\left(\mathrm{M}^{-1} \cdot \mathrm{min}^{-1}\right)\end{array} \\ & 0.56 & 4120 \\ & 0.63 & 3680 \\ 16 & 144 \\ & 9 & 256 \\ & 18 & 128 \\ & 19 & 121 \\ & 22 & 105 \\ \alpha & 30.5 & 76 \\ \epsilon & 64 & 36 \\ \alpha & 6.4 & 360 \\ \epsilon & 84 & 27 \\ \alpha & 14 & 164 \\ \epsilon & 64 & 36 \\ 1 & 25 & 91 \\ 2 & 64 & 36 \\ 3 & 8.7 & 264 \\ & 19.5 & 118 \\ & 48 & 48\end{array}$

3.9

592

26.0

89

69.0

* The $\epsilon_{420}$ value of one $\alpha$-amino group was subtracted arbitrarily from this set to permit calculation of the $\epsilon$-amino groups.

complexes determined at $\mathrm{pH} 9.5$ by procedure 2 were about $5 \%$ higher than those determined at $\mathrm{pH} 7.2$ by procedure 1. Goldfarb (1966a) reported a similar decrease in the extinction of TNP- $\alpha$-acetyl-lysine in the presence of equimolar $\mathrm{Na}_{2} \mathrm{SO}_{3}$ between $\mathrm{pH} 9.7$ and 7.2. The molar extinctions of constituent amino groups of compounds shown in Table 1 were obtained by setting the final extinction reached at pH 9.5 to that given by procedure 1 at $\mathrm{pH} 7.2$, before analysing the constituent rates.

Reagent solution. Commercial samples of TNPsulphonic acid often contained impurities that formed sulphite complexes with high extinctions at $420 \mathrm{~nm}$. Recrystallizing once as described in the Materials and Methods section usually gave $50 \%$ yield of acceptable material, but subsequent crystallizations from the mother liquor were less successful. The $1.8 \mathrm{M}$ concentration of reagent recommended may form crystals of TNP-sulphonic acid on standing. Although the crystals are easily redissolved by warming, there is the danger that a supersaturated solution will crystallize in a microsyringe or pipette. This is avoided by warming the solution to $40^{\circ} \mathrm{C}$ just before use. Alternatively, a solution of $1.1 \mathrm{~m}$-reagent [prepared by dissolving 1 part of TNP-sulphonic acid in 2 parts of water $(w / w)]$ that is stable at room temperature may be used. A check should be made, of course, that a solution of this concentration reacts to completion with the amino groups that are being determined.

Reaction of TNP-sulphonic acid with thiol groups. At pH 9.5 the reaction of TNP-sulphonic acid with the $\alpha$-amino group of L-cysteine was 25 -fold faster than with that of $S$-carboxymethyl-L-cysteine (Table 2). This rate of reaction, which is nearly equal to that with the thiol group of $N$-acetyl-Lcysteine, suggests a rapid intramolecular $\mathrm{S}$ to $\mathrm{N}$ transfer of the TNP group. Kotaki, Harada \& Yagi (1964) reported the rearrangement of $S$-TNP. cysteine to $N$-TNP-cysteine at neutral $\mathrm{pH}$, and the transfer of DNP groups from $\mathrm{S}$ to $\mathrm{N}$ is well known (Hausmann, Weisiger \& Craig, 1955; Burchfield, 1958; Wallenfels \& Streffer, 1966).

As there was no noticeable enhancement of the rate of reaction of TNP-sulphonic acid with $25 \mu \mathrm{M}$ L-alanine when $28 \mu \mathrm{M}-\mathrm{N}$-acetyl-L-cysteine was initially present (the reaction with the thiol went to completion in the first few seconds) the acceleration of $N$-trinitrophenylation by transfer of TNP-groups from $S$ under the conditions of 


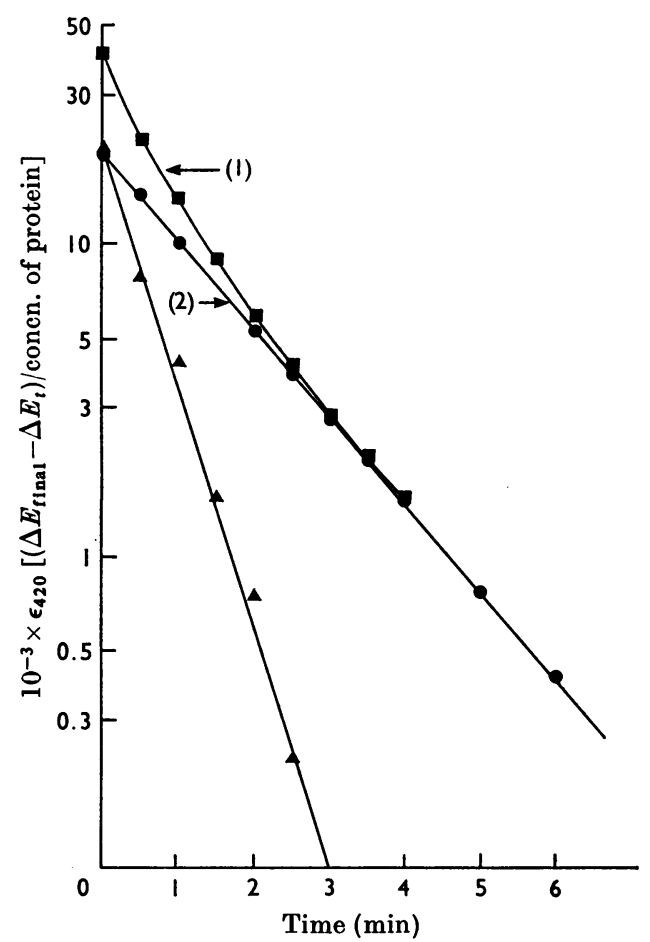

Fig. 4. Change of $E_{420}$ of ribonuclease $T_{1}$ and of transaminated ribonuclease $T_{1}$. The reactions analysed are those shown in Fig. 3. $\square$, Ribonuclease $T_{1}(1)$; $\bullet$, transaminated ribonuclease (2); $\Delta$, curve (1) minus ourve (2). The half-lives of these reactions are shown in Table 2.

procedure 2 seems to be restricted to cases in which intramolecular transfer is favourable. The occurrence of cysteine or homocysteine as the $N$-terminal residue in a protein or peptide might therefore be confirmed by the rate of its reaction with TNP-sulphonic acid. Transfers of TNP. groups from thiol to amino groups that are not $N$-terminal in proteins, may also be possible (cf. Wallenfels \& Streffer, 1966). Kotaki et al. (1964) reported $S$-TNP derivatives to be stable at neutral and alkaline $\mathrm{pH}$, provided there were no free amino groups present. The sulphite complexes of $S$-TNPgroups were found to be as stable as those of amino groups, changes in extinction being less than 5\% in $0.5 \mathrm{~h}$. The results shown in Fig. 2 indicate that a higher concentration of sulphite than that investigated (10 mM) would be necessary for the full development of the extinction associated with the $S$-TNP-sulphite complex. The difference in affinity for sulphite exhibited by TNP-thiol and TNP-amino groups (as well as the difference in $\lambda_{\max }$. of the sulphite complex) might provide a way of discriminating between these groups after reaction with

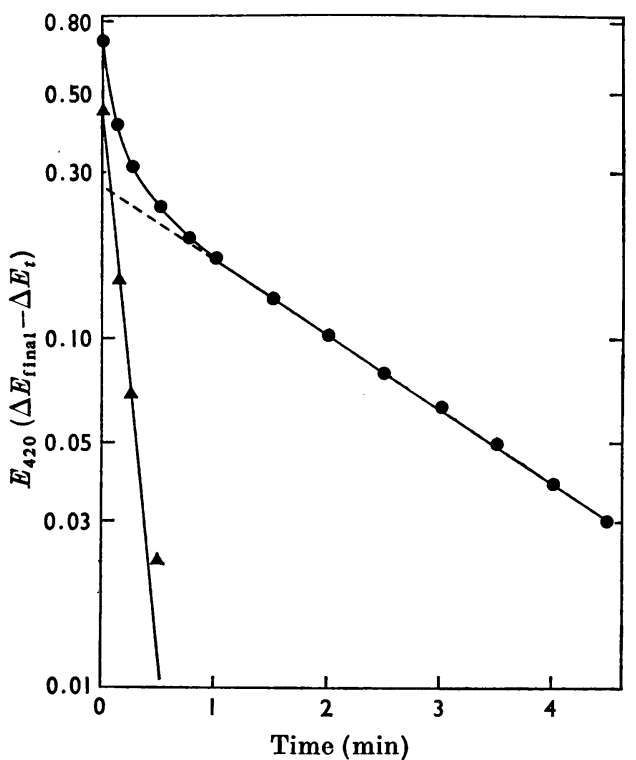

Fig. 5. Analysis of the reaction of $20 \mu \mathrm{M}-\mathrm{L}$-lysine with $18 \mathrm{~mm}$-TNP-sulphonic acid, as described in the text. The reaction was carried out according to procedure 2 . $\bullet$, $\Delta E_{\text {final }}-\Delta E_{t}$ of the original curve; -- - , extrapolated portion of the final slope of the original curve; $\Delta$, difference values of the original and extrapolated curve. The half-lives and molar extinction coefficients of the constituent amino groups are shown in Tables 1 and 2.

reagent. The extinction of a TNP-thiol group in 1 mM-sulphite is approx. $12 \%$ of that of a TNP-єamino group (Table 1). Gibbons \& Perham (1970), using the method of Habeeb (1966), reported on $E_{335}$ of TNP-thiol groups of approx. $50 \%$ that of TNP-amino groups.

Reaction of TNP-sulphonic acid with amino groups. The order of reactivity of the small-molecular-weight amines studied follows in a general way the basicity of the amino group towards protons (Fig. 6; see also Freedman \& Radda, 1968). The mechanism of nucleophilic displacement in nitroactivated aromatic systems is as yet incompletely understood, and several structures for intermedi. ates have been proposed (for reviews see Bunnett \& Zahler, 1951; Buncel, Norris \& Russell, 1968). The scatter of points shown in Fig. 6 emphasizes that these reactions are sensitive to other effects, e.g. steric, that influence the rate. The variation in the rate of reaction versus $\mathrm{pH}$ of some amino acids and derivatives fits the expected titration curve of the amino group fairly well but a marked exception is the continued increase in rate with rising $\mathbf{p H}$ in the case of aniline, even at $\mathrm{pH}$ values four units or more above its $\mathrm{pK}$ (Fig. 7). Goldfarb (1966a,b) noted that a simple mechanism based solely on the 


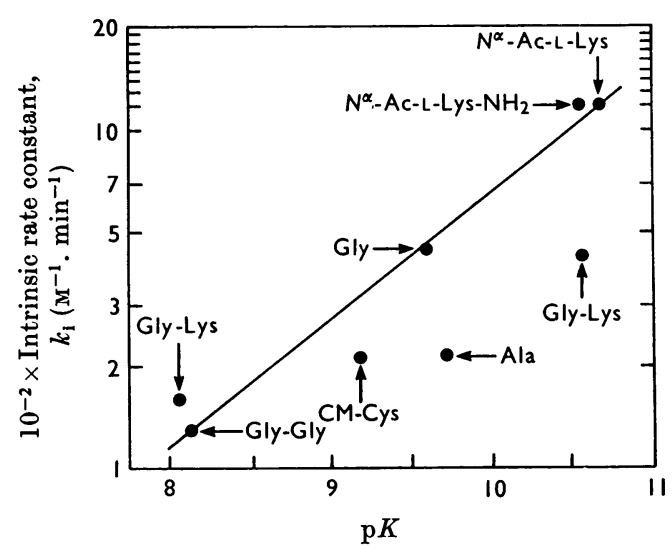

Fig. 6. Relation of basicity to the reactivity of amines towards TNP-sulphonic acid. The straight line is arbitrarily drawn through the points for glycylglycine, glycine and $N^{\alpha}$-acetyl-L-lysine and has a slope of 0.375 . Abbreviations: CM-Cys; S-carboxymethyl-L-cysteine; $N^{\alpha}$-Ac-L-Lys, $N^{\alpha}$-acetyl-L-lysine; $N^{\alpha}$-Ac-L-Lys- $\mathrm{NH}_{2}, N^{\alpha}$ acetyl-L-lysine amide.

reaction between TNP-sulphonic acid and a free amino group could not be used exclusively to explain the rates he found with glycine, di- and triglycine, or with $\epsilon$-amino groups in proteins. The intrinsic reactivities shown in Fig. 6 were calculated, however, by making such a simplifying assumption, since the deviation of experimental points from the theoretical curve for amino acid derivatives near pH 9.5 did not seem to be great (Figs. $7 a$ and $7 b$ ).

The unexpectedly small molar extinction of the sulphite complex of bis-TNP-lysine (Table 1) suggests the possibility of considerable interaction between the two TNP groups. When sulphite complexes of bis-TNP-lysine and of TNP-leucine were formed in solutions containing 10 or $50 \%(\mathrm{v} / \mathrm{v})$ ethylene glycol no change in molar extinction was observed; the interaction seems therefore not to be due to unspecific effects of the medium. The interpretation of the apparent reaction rate of lysine with TNP-sulphonic acid is discussed in the section below.

The experimental curve of the reaction of glycylL-lysine with TNP-sulphonic acid could be resolved readily into two pseudo-first-order contributions with molar extinctions of about 22000 and $19200 \mathrm{~m}^{-1} \cdot \mathrm{cm}^{-1}$. The $\mathrm{p} K$ for an amino group associated with each of these reactions could be estimated by analysing the curves produced at several $\mathbf{p H}$ values. The results are shown in Fig. $7(b)$.

The accuracy of such an analysis was tested by comparing the reaction rate of transaminated ribonuclease $T_{1}$ with that of the native enzyme; only the $\epsilon$-amino group of lysine-41 is available for reaction in the transaminated derivative. Fig. 4 shows that when the curve representing log amino groups remaining of transaminated ribonuclease $\mathrm{T}_{1}$ (curve 2) is subtracted from that of native ribonuclease $T_{1}$ (curve 1 ), the difference is a curve that is pseudo-first order. It was possible to obtain the same result from curve (1) alone within a few minutes by the trial and error method described in the Materials and Methods section.

The $\epsilon_{\mathbf{4 2 0}}$ value found for insulin (Table 1 ) is close to that expected for one $\epsilon$ - and two $\alpha$-amino groups. The finding of eight amino groups (Tables 1 and 2) for ribonuclease $A$ agrees with the results obtained by Freedman \& Radda (1968). Ribonuclease A is known to possess a binding site for a single bivalent anion that is thought to be near the active site (Kartha, Bello \& Harker, 1967). The failure to observe reaction with all 11 of the known amino groups may therefore represent a shielding of the negatively charged reagent by a phosphate or borate ion at the active site. The possibility of complete reaction with TNP-sulphonic acid cannot be discounted, however, in the absence of a chemical analysis of the TNP-ribonuclease derivative. The interaction of TNP groups with one another (as in the case of lysine) or with aromatic side chains of the protein may give rise to hypochromicity of TNP-amino group-sulphite complexes in the protein.

In simple peptide systems it may be possible to determine the $\mathrm{p} K_{\mathrm{a}}$ values of several amino groups with as little as $50 \mathrm{nmol}$ samples by using procedure 2 at several pH values (see Figs. $7 a$ and $7 b$ ). The procedure is rapid and may also be used to determine apparent $\mathrm{p} K_{\mathrm{a}}$ values of amino groups in proteins, provided that changes in $\mathrm{pH}$ do not affect protein conformation.

Precautions. The reaction of TNP-sulphonic acid with L-lysine provides a model that exhibits many of the difficulties inherent in the use of a chromophoric reagent for obtaining reliable information about the nature and number of nucleophilic groups in proteins. The biphasic semi-logarithmic plot of the reaction shown in Fig. 5 yields component reactions that together fit the experimental points reasonably well. Yet from Scheme 1 it is clear that the changes in extinction occurring during trinitrophenylation of L-lysine are much more complex than could have been adduced from an examination of the curve. Further, the 'component reactions' shown in Fig. 5 reflect only approximately the trinitrophenylation of the $\epsilon$ - and the $\alpha$-amino groups. The molar extinction changes shown in Scheme 1 were assigned by assuming typical values (for the initial reactions) for $\alpha$-and $\epsilon$-amino groups, and by subtraction of these values from the molar extinction coefficient found for L-lysine (Table 1). 

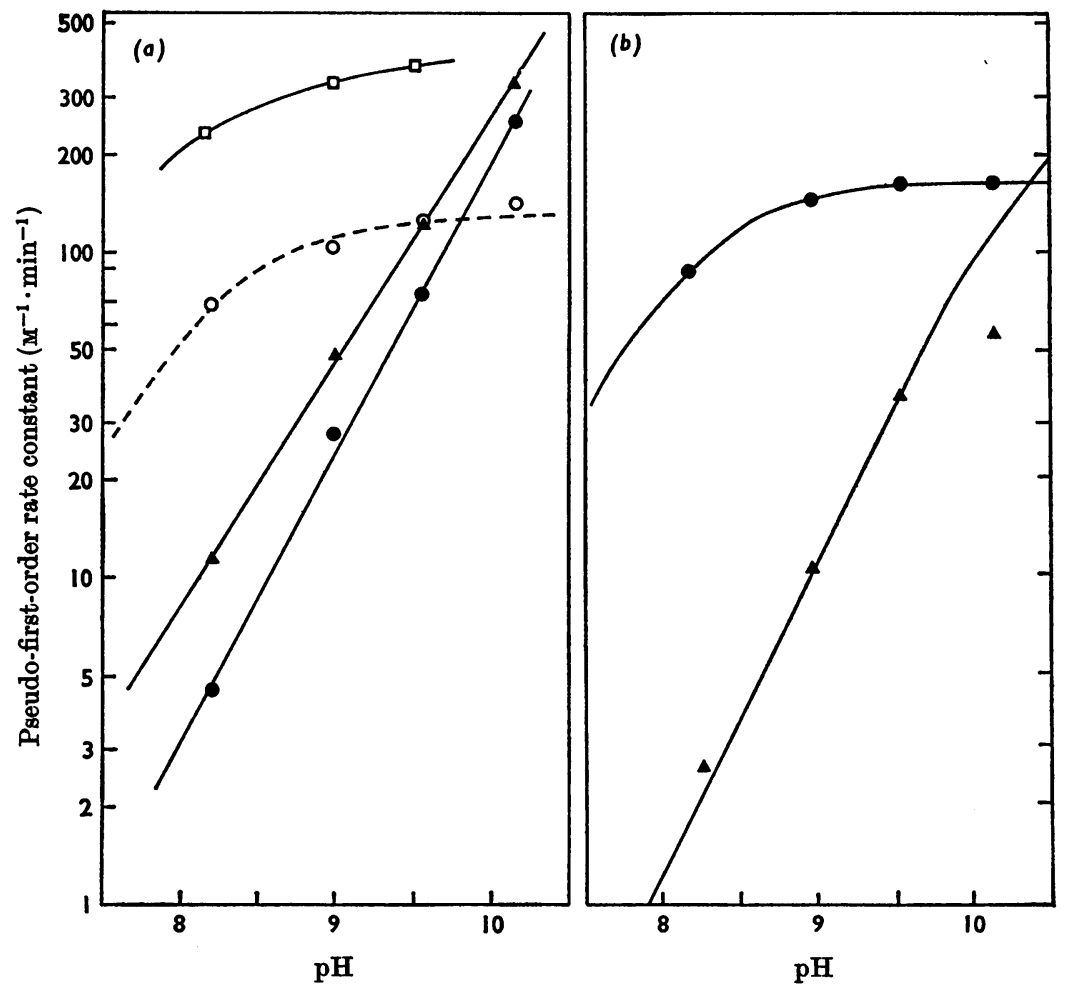

Fig. 7. Reactivity of various amines towards TNP-sulphonic acid at $20^{\circ} \mathrm{C}$ as a function of $\mathrm{pH}$. The reactions were carried out according to procedure 2 , in $0.1 \mathrm{~m}$-borate buffer that was adjusted with $10 \mathrm{M}-\mathrm{HCl}$ or $10 \mathrm{M}-\mathrm{NaOH}$ to give the $\mathrm{pH}$ value shown. $(a) \square$, Aniline, $\mathrm{p} K 4.6 ; \mathrm{O}$, glycylglycine, $\mathrm{p} K_{2} 8.13 ; \Delta, N^{\alpha}$-acetyl-L-lysine amide, $\mathrm{p} K 10.53$; $\bullet, N^{\alpha}$-acetyl-L-lysine, $\mathrm{p} K_{2}$ 10.7. - - - Theoretical curve for an amino group with $\mathrm{p} K 8.13$ and an intrinsic rate constant of $130 \mathrm{M}^{-1} \cdot \mathrm{min}^{-1}$. (b) Constituent reactions of the amino groups of glycyl-L-lysine, $\mathrm{p} K_{2}$ 8.05, $\mathrm{p} K_{3}$ 10.55, determined by the procedure outlined in the Materials and Methods section. $\bullet$, Faster-reacting group. The line through these points is that of the theoretical curve for an amino group with $\mathrm{p} K 8.05$ and an intrinsic rate constant of $162 \mathrm{M}^{-1} \cdot \mathrm{min}^{-1}$. $\Lambda$, Slower-reacting group. The line shows the theoretical curve for an amino group with $\mathrm{p} K 10.55$ and an intrinsic rate constant of $425 \mathrm{M}^{-1} \cdot \mathrm{min}^{-1}$.

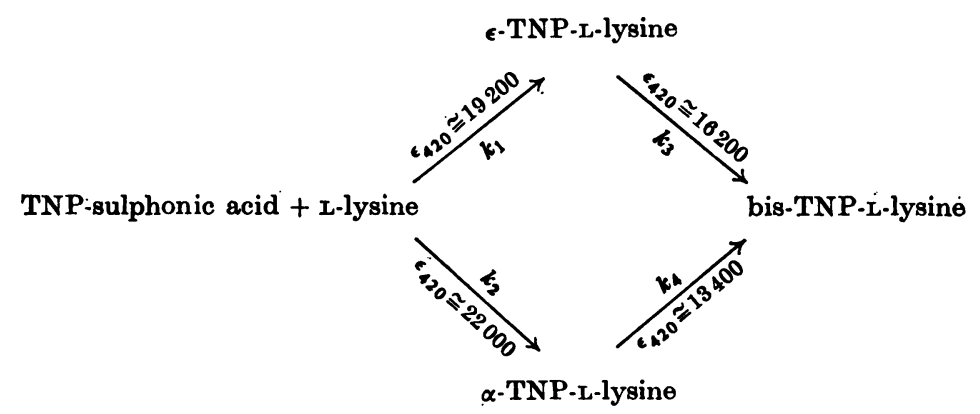

Scheme 1 .

A comparison of the apparent rates of reaction of the constituent $\alpha$-and $\epsilon$-amino groups of $L$-lysine, with those of glycyl-L-lysine, ribonuclease $\mathbf{T}_{1}$,
$N$-acetyl-L-lysine, and with the appropriate model $\alpha$-amino groups (Table 2), shows that the $\alpha$-amino group reaction is faster than expected, whereas that 
with the $\epsilon$-amino group is slower. From Scheme 1 it follows that the rate of change in $E_{420}$ at any given time will be given by:

$$
\begin{aligned}
\frac{\mathrm{d} E_{420}}{\mathrm{~d} t}= & 1.92 k_{1}[\mathrm{~L}-\mathrm{ly} \sin \Theta]+2.2 k_{2}[\mathrm{~L}-\mathrm{ly} \sin \Theta] \\
& +1.62 k_{3}[\epsilon-\mathrm{TNP}-\mathrm{L}-\mathrm{lysin} \Theta] \\
& +1.34 k_{4}[\alpha-\mathrm{TNP}-\mathrm{L}-\mathrm{ly} \sin \Theta]
\end{aligned}
$$

where concentrations are expressed in units of $10^{-4} \mathrm{M}$. Assuming $k_{1}=k_{4}$ and $k_{2}=k_{3}$ and that $k_{1}$ and $k_{2}$ are not very greatly different in value it may be observed that: (1) the reaction of TNP. sulphonic acid with L-lysine (whether with an $\epsilon$ or with an $\alpha$-amino group) will in the beginning give a greater change in extinction for each reacting molecule of TNP-sulphonic acid than later. (2) The reaction of TNP-sulphonic acid with a monosubstituted derivative of L-lysine $\left(k_{3}\right.$ and $\left.k_{4}\right)$ will cause a smaller change in extinction relative to the actual rate of trinitrophenylation. The early reactions therefore will contribute to the appearance of a faster-reacting kinetic set than actually exists, whereas the later reactions will give the appearance of a lower rate of trinitrophenylation than that which is taking place. Extrapolation of the straight portion of the $\log \left(\Delta E_{\text {final }}-\Delta E_{t}\right)$ curve, as shown in Fig. 5, gives a value that cannot be assigned as the molar extinction of any constituent amino group.

The plot of the reaction in Fig. 5 is shown to about $95 \%$ completion. When the extinction changes are plotted further, the apparent rate of reaction begins to increase, and produces a semi-logarithmic plot of $\Delta E_{\text {final }}-\Delta E_{t}$ that is sigmoid. The fact that this does not appear until the very final stage of the reaction emphasizes the desirability of following reactions to completion, when possible, before analysing results.

The studies made here suggest that the requirements for accurate analytical data are stringent (cf. also Singer, 1967). If a 'kinetic set' of reacting amino groups, derived from analysis of an experimental curve, is to correspond with specific amino groups in a protein, each amino group must react independently with the reagent and there must be no hypochromic or hyperchromic interactions. If reacting groups are buried within the protein, any changes in conformation that occur during trinitrophenylation, e.g. as a result of the introduction of hydrophobic TNP-groups, may easily increase or decrease the rate of reaction of TNP-sulphonic acid with other partly buried residues. Accurate quantitative analysis of experimental curves in such cases is almost certain to be impracticable, owing to the distribution of modified species at any given time. The 'kinetic sets' obtained by analysis of such curves cannot accurately correspond to groups in the protein.
The interpretation of absorption results should, therefore be cautious, and a reactive group that has been found by kinetic analysis ought if possible to be shown, by measurement at several values of $\mathrm{pH}$, to change its rate of reaction in a way that is consistent with that group's possessing a fixed $\mathrm{p} K$ value (Figs. $7 a$ and $7 b$ ). If this cannot be done, characterization of the group by peptide mapping (Anderson \& Perham, 1970; Hartley, 1970) or by another chemical means would seem necessary.

The concentration of sulphite ( $1 \mathrm{~mm})$ used in procedure 2 was found not to affect the rate of reaction of TNP-sulphonic acid with simple amines. However, the cleavage of disulphide bridges by sulphite (Cecil \& McPhee, 1955) is a possibility that should not be ignored. A simple check consists of a comparison of the results obtained in the presence of sulphite with the results from an experiment in which no sulphite has been added. The value for total amino groups obtained by procedure 1 , in which sulphite is added only after the reaction with reagent is complete is an approximate guide. However, sulphite cannot be entirely eliminated from the system as it is generated during the course of the reaction.

I am most grateful to Dr H. B. F. Dixon for many helpful discussions and for his invaluable aid in the preparation of this manuscript. I also thank $\mathrm{Dr} I$. Gibbons for discussions and for carrying out some of the amino acid analyses. I am happy to acknowledge support from King's College, Cambridge, and from the Science Research Council.

\section{REFERENCES}

Anderson, P. J. \& Perham, R. N. (1970). Biochem. J. 117, 291.

Buncel, E., Norris, A. R. \& Russell, K. E. (1968). Q. Rev. chem. Soc. 22, 123.

Bunnett, J. F. \& Zahler, R. E. (1951). Chem. Rev. 49, 273.

Burchfield, H. P. (1958). Nature, Lond., 181, 49.

Cecil, R. \& McPhee, J. R. (1955). Biochem. J. 60, 496.

Clarke, H. T. (1932). J. biol. Chem. 97, 235.

Dixon, H. B. F. (1967). Biochem. J. 103, 38 P.

Ellman, G. L. (1959). Archs Biochem. Biophys. 82, 70.

Fields, R. \& Dixon, H. B. F. (1971). Biochem.J.121, 587.

Fields, R., Dixon, H. B. F., Law, G. R. \& Yui, C. (1971). Biochem. J. 121, 591.

Freedman, R. B. \& Radda, G. K. (1968). Biochem. J. 108, 383.

Gibbons, I. \& Perham, R. N. (1970). Biochem. J. 116, 843.

Goldfarb, A. R. (1966a). Biochemistry, Easton, 5, 2570.

Goldfarb, A. R. (1966b). Biochemistry, Easton, 5, 2574.

Habeeb, A. F. S. A. (1966). Analyt. Biochem. 14, 328.

Hartley, B. S. (1970). Biochem. J. 119, 805.

Hausmann, W., Weisiger, S. R. \& Craig, L. C. (1955). J. Am. chem. Soc. 77, 723.

Humphrey, R. E., Ward, M. H. \& Hinze, W. (1970). Analyt. Chem. 42, 698. 
Kartha, G., Bello, J. \& Harker, D. (1967). Nature, Lond., Satake, K., Okuyama, T., Ohashi, M. \& Shinoda, T. (1960). 213, 862.

J. Biochem., Tokyo, 47, 654.

Kotaki, A., Harada, M. \& Yagi, K. (1964). J. Biochem., Sela, M. \& Anfinsen, C. B. (1957). Biochim. biophys. Tokyo, 55, 553.

Acta, 24, 229.

Okuyama, T. \& Satake, K. (1960). J. Biochem., Tokyo, Singer, S. J. (1967). Adv. Protein Chem. 22, 1. 47, 454.

Wallenfels, K. \& Streffer, C. (1966). Biochem. Z.346, 119. 